\title{
Identification of Quantitative Trait Loci for Mesocotyl Length of Rice Seedling in Different Sowing Depths using Genome-Wide Association Study
}

\section{Chuanhang Yang}

Rice Researcher Institution, Sichuan Agricultural Univesity

Hao Wang

Sichuan Agricultural University

Xiaojuan Tang

Sichuan Agricultural University

Qing Guo

Sichuan Agricultural University

\section{Youhui Li}

Sichuan Agricultural University

Yi Liu

Sichuan Agricultural University

\section{Xiuxiu Li}

Chinese Academy of Sciences

\section{Peng Qin}

Sichuan Agricultural University

\section{Bin Tu}

Sichuan Agricultural University

\section{Weilan Chen}

Sichuan Agricultural University

Hua Yuan

Sichuan Agricultural University

\section{Bingtian Ma}

Sichuan Agricultural University

\section{Chengzhi Liang}

Chinese Academy of Sciences

\section{Shigui Li}

Sichuan Agricultural University

\section{Yuping Wang ( $\nabla$ wypscwj@126.com )}

Rice Research Institute, Sichuan Agricultural University https://orcid.org/0000-0002-8639-6637 
Original article

Keywords: Rice seedling, Mesocotyl length, GWAS, Quantitative trait locus, Sowing depth

Posted Date: November 6th, 2020

DOl: https://doi.org/10.21203/rs.3.rs-101899/v1

License: (c) (1) This work is licensed under a Creative Commons Attribution 4.0 International License. Read Full License 


\section{Abstract}

Background: The mesocotyl length of rice seedling is a complex trait associated with the ability of seedling emerging and controlled by quantitative trait loci (QTLs). Genome-wide association study (GWAS) is a high-through QTLs mapping method and widely used to identify QTLs in the field of plant genetics.

Results: A series of rice accessions including 290 varieties from all over the world, which harbored widely genetic background, were finished whole genome re-sequencing and investigated the mesocotyl length of rice seedlings in three different sowing depths $(0 \mathrm{~cm}, 4 \mathrm{~cm}$ and $6 \mathrm{~cm})$. The analysis of coefficient of variation $(\mathrm{CV})$ showed that the rice mesocotyl length variability in the three different treatments with $0 \mathrm{~cm}$, $4 \mathrm{~cm}$ and $6 \mathrm{~cm}$ sand culture are $114.43 \%, 146.65 \%$ and $152.55 \%$, respectively. The mesocotyl length of the three groups are positively correlated with each other, indicating that the variation trend is consistent despite the differences in experimental treatment conditions, which led to the different performances of the mesocotyl lengths of the same accession. After GWAS, we found There are 922 single nucleotide polymorphism (SNP) makers associated with rice seedling mesocotyl length across 20 QTL regions and covered 1246 genes.

Conclusions: In this study, we found that there are 922 SNP makers associated with rice seedling mesocotyl length across 20 QTL regions and covered 1246 genes. And we detected 23 candidate genes which maybe have effect to the mesocotyl length of rice seedling at different sowing depths

\section{Background}

Rice is one of the most important crops in the world. In traditional rice cultivation, rice is sprouted first and the fully germinated seedling is then replanted into a permanent location, termed as seedling transplanting. Compared to seedling transplanting, direct seedling is a simplified cultivation technology that has the advantages of saving labor and energy, environmental protection and higher cost efficiency. Although previously direct seedling is associated with low seedling emergence rate, difficulty in weed control and weak lodging resistance, it becomes more and more popular with significant developments in recent years(Farooq et al. 2011; Kumar and Ladha 2011; Shahi and RAU 2015; Soriano et al. 2018). For instance, in the USA, Italy and Australia, almost all rice is direct seeded(Kumar and Ladha 2011).. In Asia, more than $50 \%$ of rice is direct seeded in Sri Lanka, Malaysia and some parts of India; and the directseeded rice fields are also increased rapidly in China, Philippines, South Korea, Japan, Thailand and several other countries recently(Chen et al. 2018; S Marasini 2016; Sansen et al. 2019; Weerakoon et al. 2011).

Direct seedling of rice is roughly divided into paddy field direct seedling and dryland direct seedling, in which dryland direct seedling rice is particularly important for arid and semi-arid areas. Rice cultivation with direct seedling is greatly affected by sowing depth. Although increasing sowing depth within a certain range increased drought resistance and lodging resistance of rice, too deep seedling significantly 
reduced seedling emergence. Previous studies have shown The length of mesocotyl at different sowing depths is positively correlated to the rice seedling emergence rate(Dilday et al. 1990; Ohno et al. 2018). Moreover, the rice mesocotyl length is also essential for its capacity to unearth and early vigor(Allan et al. 1961; Purchase et al. 1992). However, most current rice accessions are semi-dwarf accessions with short mesocotyl that showed low emergence rate when planted deeply (Turner et al. 1982; Wu et al. 2015).

Rice mesocotyl length is determined by both environmental and genetic factors. Environmental factors, such as light condition, temperature, soil moisture condition, oxygen concentration, sowing depth and exogenous hormones, can affect mesocotyl elongation(Gao et al. 2012; Hu et al. 2010; Watanabe et al. 2001; Yalan 2016). Genetic analysis showed that the length of rice mesocotyl was controlled by multiple genes, which varied from accession to accession but could be inherited stably(Redoña and Mackill 1996). There are many rice mesocotyl length quantitative trait loci which are distributed on 12 chromosomes identified by different mapping populations(Lee et al. 2012,2017; Wu et al. 2015; Yalan 2016; Zhao et al. 2018). Hyun-Sook Lee et al(Lee et al. 2012) detected 7 QTLs associated with mesocotyl length on rice chromosomes 1, 3, 7, 9 and 12 using restriction fragment length polymorphism(RELP) molecular makers by BILs population which was constructed by hybridization of rice accessions Nipponbare and Kasalath. Ya-lan Li et al(Yalan 2016) constructed the rice F3 population from the cross between the parental lines of 'Zhaxima' and 'Hanhui No. 3', and detected 11 QTLs related to rice mesocotyl length on chromosomes $1,3,6,8,11$ and 12 by BSA. With the development and application of NGS and GWAS, more and more loci related to rice mesocotyl length were found precisely: Jing-hong Wu et al(Wu et al. 2015) in a GWAS research detected 7 rice mesocotyl length QTLs distributing chromosome 1,3,4,5,6,9,10 including 36 genes; Yan Zhao et al(Zhao et al. 2018) used GWAS to associate the re- sequencing data of 621 rice cultivars and the seedling mesocotyl length in the $10 \mathrm{~cm}$ sand-culture condition and detected 13 QTLs controlling rice mesocotyl length predicted 21 candidate genes distributing rice chromosome 2, 3, 4, 7, 8, 9,10 and 11 .

\section{Results}

\section{The variation of rice mesocotyl length}

The analysis of CV (Table 1 \& Table S1) showed that the rice mesocotyl length variability in the three different treatments with $0 \mathrm{~cm}, 4 \mathrm{~cm}$ and $6 \mathrm{~cm}$ sand culture are $114.43 \%, 146.65 \%$ and $152.55 \%$, respectively. While most mesocotyl lengths in the three groups are in $0.00 \sim 0.50 \mathrm{~cm}$; some are above $1.00 \mathrm{~cm}$. There are 10 (the least) and 20 (the most) rice accessions whose mesocotyl lengths are in the range of $1.00 \sim 3.00 \mathrm{~cm}$ when cultured with a sowing depth of $4 \mathrm{~cm}$ and $6 \mathrm{~cm}$, respectively (Fig. 1). For each rice accession, the difference in mesocotyl lengths at different sowing depths is within $1 \mathrm{~cm}$. The analysis of variance (ANOVA) showed that there is significant difference in the mesocotyl length of 290 rice accessions in the three groups (Table 2) Although there was difference in the mesocotyl length for the same accession when cultured with different sowing depths, the trend of change in mesocotyl length for each accession is highly correlated to the sowing depths. 
The experiments results show that there is extensive variation but similar variation pattern for the mesocotyl length of rice seedling in the 290 rice accessions. According to the experimental data, the coefficient of variation (CV) of the mesocotyl lengths in experimental rice population is large and the averages of dispersion is high. This means that the experimental rice population which we used in this study have huge genetic difference and broad representation. The mesocotyl length in the most of the experimental accessions is short. And the variation of mesocotyl length is relatively slight when the treatment condition is $4 \mathrm{~cm}$. It may be because that the depth of sand was shallow, and the seedling growth may be affected by light, thus the mesocotyl elongation was limited(Feng et al. 2017; Gao et al. 2011; Kondo et al. 2010; Yalan 2016). The mesocotyl length of the three groups are positively correlated with each other, indicating that the variation trend is consistent despite the differences in experimental treatment conditions, which led to the different performances of the mesocotyl lengths of the same accession.

\section{Population structure analysis and whole genome resequencing of 290 rice accessions}

There are 115 indica rice accessions, 133 japonica rice accessions and 42 Indica japonica intermediate types in the whole 290 experimental rice accessions from all over the world (Table S2). This germplasm resource population can represent two subspecies of rice and have rich genetic background, so this population is suitable for GWAS of mesocotyl length traits in rice seedlings. The average sequencing depth in the whole genome resequencing was $10 \times$ for the 290 rice accessions and developed 1095430 SNPs genotypes. The MSU database(Xiong et al. 2017) http://rice.plantbiology.msu.edu was used to annotate the identified variations. It is the molecular basic for GWAS.

\section{Detection of SNPs associated with mesocotyl length in rice seedling}

The EMMAX software was used to analyze the correlation between the rice seedling mesocotyl length data and the SNP data developed by resequencing in 290 rice accessions, and 922 SNPs significantly was found to be correlated with the mesocotyl length were identified (Table 3). 85, 48 and 186 SNPs (-log ( $P$ value) $\geq 8.0$ ) were identified when cultured at dark with $0 \mathrm{~cm}$ sand, $4 \mathrm{~cm}$ sand and $6 \mathrm{~cm}$ sand, respectively. 6 QTL regions across chromosomes 1, 4, 8 and 10 under $0 \mathrm{~cm}$ sand dark culture condition; 2 QTL regions across chromosomes 7 and 8 under $4 \mathrm{~cm}$ sand culture condition and 4 QTL regions across chromosomes 1, 6, 7 and 9 were the distribution of associated SNP makers. Because Bonferroni correction can be conservative, it may result in the probability of producing false negatives. Therefore, we identified the SNP makers associated with rice mesocotyl length again with the -log(P value $) \geq 7.0$. And under $4 \mathrm{~cm}$ and $6 \mathrm{~cm}$ sand culture conditions, we newly identified 172 and 428 SNP makers, respectively. 172 SNP makers were across 2 QTL regions distributed in chromosomes 6 and 9. 428 SNP makers were in across 5 QTL regions distributed in chromosomes 1, 6, 7 and 12. The Manhattan plots of p-values analyzed the SNP makers associated with rice seedling mesocotyl length showed the visible loci in chromosomes (Fig. 2\&3).

\section{The comparative analysis of associated SNP makers in three different culture conditions}


There were total 8 chromosomes were identified the SNP makers associated with rice seedling mesocotyl length in three different culture conditions. And there were identified 85, 220 and 614 SNP makers (- $\log (P$ value) $\geq 7.0$ ) associations under $0 \mathrm{~cm}, 4 \mathrm{~cm}$ and $6 \mathrm{~cm}$ sand culture conditions, respectively. And the comparative analysis of the QTL regions in three different culture conditions showed that there was no overlap among $0 \mathrm{~cm}, 4 \mathrm{~cm}$ or $6 \mathrm{~cm}$ sand culture conditions, but there were three overlap regions between $4 \mathrm{~cm}$ and $6 \mathrm{~cm}$ sand culture conditions. The overlap regions were distributed in chromosomes 6,7 and 9 . The length of regions was $297.472 \mathrm{~Kb} \otimes 253.012 \mathrm{~Kb} \otimes 312.368 \mathrm{~Kb}$, respectively. The repetitive SNP maker number in three overlap regions was 112,9 and 5 , respectively.

These results showed that there were wide differences in SNP makers associated with rice seedling mesocotyl length among three culture conditions. For each culture conditions there were many specific SNP makers, although there were some overlapped SNP makers between $4 \mathrm{~cm}$ and $6 \mathrm{~cm}$ sand culture conditions. It indicates that there are multiple genes controlling rice seedling mesocotyl length, and rice seedling mesocotyl length is sensitive to environmental influences.

\section{Identification of candidate genes for seedling mesocotyl length}

There are 922 SNP makers associated with rice seedling mesocotyl length across 20 QTL regions and covered 1246 genes. There are little genes known the functions among these genes, and most of these genes are annotated as the unknown expressed protein or putative protein in http://rice.plantbiology.msu.edu (Xiong et al. 2017). And some of these genes are annotated as the regulated genes of growth and development or transcription factors.

For $6 \mathrm{~cm}$ sand culture condition, there were 7 QTL regions associated with seedling mesocotyl length. And total 11 SNP makers was located in $q M L_{6 \mathrm{~cm}} 6-1$ covered $1687.342 \mathrm{~kb}(6610901-8298243)$ region in Chr6. The $q M L_{6 \mathrm{~cm}}$ 6-1 includes 2 GDSL-like lipase/acylhydrolase genes(LOC_Os06g12410, LOC_Os06g14630)and 1 growth regulator related protein gene(LOC_Os06g13215). Moreover, there were 173 SNP makers located in $q M L_{6 \mathrm{~cm}} 6$-2(Chr6:27582320-28071845) covered $489.525 \mathrm{~kb}$ region including a MYB gene(LOC_Os12g39640) and 2 Auxin-responsive Aux/IAA gene family member genes OsIAA30(LOC_Os12g40890), OsIAA3LLOC_Os12g409000.

For $0 \mathrm{~cm}$ sand dark culture condition, 6 QTL regions were identified associated with seedling mesocotyl length. In $q M L_{O c m} 8$, there are 28 SNP makers located in $363.334 \mathrm{~kb}$ region (Chr8:25706134-26069468) covered 66 candidate genes including an auxin response factor OsARF21(LOC_Os08g40900). And there were 2 OsWAK receptor-like protein kinase genes (OsWAK34, OsWAK33) found in $q M L_{O c m} 4$ and 3(OsWAK95, OsWAK96, OsWAK97) in $q M L_{\text {Ocm }}$ 10-1. Furthermore in $q M L_{O_{c m}} 10-1$, an OsWAK receptor-like cytoplasmic kinase gene(OsWAK98, LOC_Os10g02360) and an oxidoreductase, aldo/keto reductase family protein gene(LOC_Os10g02380) were identified.

GDSL-like lipase/acylhydrolase genes are mainly expressed in the root of rice seedlings and possibly regulate the cell elongation as well as extracellular matrix. EGR1 with a GDSL-motif domain was as a negative regulator of coleoptile elongation in the context of recent findings on the impact of JA on light 
signaling(GER1, a GDSL Motif-Encoding Gene from Rice is a Novel Early Light- and Jasmonate-Induced Gene). The main function of MYB family transcription factors was the regulator of secondary metabolism and Cellular morphogenesis, and MYB family transcription factors were expressed in different tissues and organs at different development stages of rice(Hande et al. 2017; Slabaugh et al. 2011). OsSAUR25 and OSSAUR26 are the member of the SUAR gene family which is as part of the primary auxin response in plants and involved in plant growth by regulating auxin synthesis and transport(Xihua et al. 2017; Chen et al. 2014; Kant and Rothstein 2009; Niek et al. 2018; Spartz et al. 2012). OsIAA30 and OSIAA3 are expressed in different tissues and organs at different development stages of rice especially highly expressed in the root of seedling, as reported that these two genes take part in the signal transduction of auxin(Jie et al. 2018; Nakamura et al. 2010). The genes in the wallassociated kinase (WAK) gene family have important function in plant growth and development such as signal transduction, Resistance of pathogens, response to mineral element and cell elongation(Shibo Zhang 2005). In rice, the genes of OsWAK receptor-like protein kinase are expressed in different tissues and organs at different development stages, OsWAK1 is part in the defense of plant disease(Li et al. 2009); and OsWAK11 is regulated by Aluminum (Al), Copper (Cu) and Sodium (Na)(Wei et al. 2014). But the function of six OsWAK genes in our associated QTL regions is not clear. And aldo/keto oxidoreductase gene is expressed the leaf and radicle, part in the regulation of cell elongation(Hur et al. 2009).

Taken together, based on our results and previous publications, we detected 23 candidate genes which maybe have effect to the mesocotyl length of rice seedling at different sowing depths(Table 4)(Kutschera and Wang 2016; Huizhen et al. 2018; Qi et al. 2013; Wang et al. 2007).

\section{Discussion}

\section{The advantages and disadvantages of GWAS of rice seedling mesocotyl length trait}

GWAS, based on whole genome re-sequencing, is the crucial method to identify locus of target trait due to its high throughput and resolution. The resolution of GWAS can even reach the single gene level. However, GWAS requires a large sample size and sufficient sequencing depth to ensure the accuracy of the analysis. In this study, we re-sequenced 290 rice accessions with the $\sim 10 \mathrm{X}$ sequencing depth. The resequencing materials in this study were rich in genetic background from all over the world which covered both indica and japonica (about 1:1). For the sample size, sequencing depth and genetic variation, our materials meet the requirements of GWAS. But on the other hand, the regulation of rice seedling mesocotyl length is complex because it is controlled by multiple genes and is sensitive to environmental factors. For these reasons, we still may have missed some SNP makers associated with rice seedling mesocotyl length.

\section{The comparative analysis of QTLs associated the trait of rice seedling mesocotyl length}

In the previous study, researchers had identified some QTLs and genes associated with the trait of rice seedling mesocotyl length distributed in all over 12 chromosomes of rice(Lee et al. 2012,2017; Wu et al. 
2015; Yalan 2016; Zhao et al. 2018) (Table 3). In this study, we had identified 922 SNP makers association covered 20 QTL regions with 1246 genes across 8 chromosomes including Chr1, 4, 6, 7, 8, 9, 10 and 12. And in our detected QTL regions, 4 QTLs $\left(q M L_{6 c m} 1-3, q M L_{4 c m} 9, q M L_{6 c m} 9, q M L_{6 c m} 12-2\right)$ are adjacent or partially overlapping with 3 QTLs ( $q M e l-1, q M e l-9, q M e l-12)$ which had been reported in the study of Hyun-Sook Lee et al(Lee et al. 2012) (Table 3). 8 QTLs ( $q M L_{0 \mathrm{~cm}} 1-1, q M L_{0 \mathrm{~cm}} 1-2, q M L_{4 \mathrm{~cm}} 6$, $\left.q M L_{4 c m} 7, q M L_{6 c m} 6-1, q M L_{6 c m} 6-2, q M L_{6 c m} 7-2, q M L_{6 c m} 12-2\right)$ in Chr1, 6, 7 and 12 are adjacent or partially overlapped with 6 QTLs and in the study of Ya-lan Li et al(Li 2016). There are 6 QTLs $\left(q M L_{o c m}{ }^{1-2 \text {, }}\right.$ $\left.q M L_{0 c m} 4, q M L_{4 c m} 6, q M L_{6 c m} 6-2, q M L_{4 c m} 9, q M L_{6 c m} 9\right)$ in our study being adjacent with the QTLs in Jinghong Wu's(Wu et al. 2015) study. And $q M L_{6 c m} 1-3$ in the Chr1 is adjacent with $G Y 1$ (Xiong et al. 2017), a gene functions at the initial step of jasmonic acid (JA) biosynthesis to repress mesocotyl and coleoptile elongation in etiolated rice seedlings, cloned in rice accession Kasalath (Table 3).

In this study, we have detected 23 candidate genes possibly associated with the trait of rice seedling mesocotyl length. These 23 candidate genes cover 6 chromosomes including Chr4, 6, 7, 8, 10, and 12. In the Chr7, we have identified a QTL $\left(q M L_{6 c m} 7-1\right)$ which is adjacent with $q F M L 7-2$ and overlap $q F M L 7-3$, two QTLs identified by Yan Zhao(Zhao et al. 2018). And that both we have predicated a gene LOC_Os07g24010, a functionally unknown hypothetical protein (Table 4).

There are 8 of 20 QTLs associated with the trait of rice seedling mesocotyl length in our study that are newly identified and no intersection nor adjacent with reported QTLs. These 8 newly identified QTLs may be some marginal effects specific locus in our population. In our study, the candidate genes predicted to be associated with the trait of rice seedling mesocotyl length had a low repetition rate with the results predicted by previous study. On the one hand, that's because of the difference in experimental population, on the other hand, the trait of rice seedling mesocotyl length is susceptible to environmental influences. Thus, it is complex to investigate the QTLs controlled rice seedling mesocotyl length.

\section{Breeding application}

Rice direct sowing maybe become the trend of rice cultivation techniques for saving the cost of labor and reducing the working procedure. Rice seedling mesocotyl length is an important trait for rice direct sowing. In the process of rice direct sowing, the longer mesocotyl in rice seedling, the higher rate of seedling emergence is. In our study, we had identified 20 QTLs and a series of genes associated with the trait of rice seedling mesocotyl length in natural population by GWAS. And some of these QTLs are overlap or adjacent with previous reported study, that indicates the credibility of our results. For breeders, eight newly identified QTLs in our study may facilitate to develop new strategies for improving the ability of rice direct sowing.

\section{Conclusion}

In our study, we had cultivated 290 rice accessions in three different conditions $(0 \mathrm{~cm}$ dark; $4 \mathrm{~cm}$ sand and $6 \mathrm{~cm}$ sand growing conditions). In three growth conditions, we had detected 14, 10 and 20 rice accessions which have more than $1 \mathrm{~cm}$ rice seedling mesocotyl, respectively. And there are total 10 rice 
accessions having more than $1 \mathrm{~cm}$ seedling mesocotyl in all the culture conditions. Moreover, we had identified 922 SNP makers associated with the trait of rice seedling mesocotyl length and hit 1246 genes. After bioinformatic analysis, we had got 23 candidate genes. It would be a reference for the next work of gene mapping and cloning.

\section{Methods}

\section{Planting and growth condition}

290 rice accessions (Table.S2) were used as GWAS population, in which 220 accessions were selected from International Rice Research Institute (IRRI); and the other 70 accessions were from Institute of Genetics and Developmental Biology, Chinese Academy of Sciences. These accessions are from all over the world and have the extensive genetic background. The experiments were carried out in Sichuan Agricultural University, Chengdu, China. 100 healthy ripe seeds were selected for each accession and sterilized with $3 \%$ hydrogen peroxide. The seeds were then put into $28^{\circ} \mathrm{C}$ water for 36 hours to sprout. Each accession was transported in three float tray holes (20 sprouting seeds/ hole). For each accession, there were three individual float tray holes and every hole covered with different depths of sand $(0 \mathrm{~cm}$, $4 \mathrm{~cm}, 6 \mathrm{~cm}$ ). The float trays were placed in constant temperature rooms at $22{ }^{\circ} \mathrm{C}$ with $65 \%$ air humidity for 12 days. And the $0 \mathrm{~cm}$ float trays are shading treatment; the others sapper-light conditions are $14 \mathrm{~h}$ light and $10 \mathrm{~h}$ dark in a single day.

\section{Evaluation of rice mesocotyl length}

After 12 days of cultivation, the length of the mesocotyl was measured with the millimeter scale. 20 seedlings were measured in each single float tray hole.

\section{Whole genome sequencing, SNP calling and Genotyping}

Whole genome re-sequencing was performed for 290 rice accessions by using Illumina hi-seq sequencing system. Briefly, paired-end sequencing with $125-150$ bp reading length ( $10 \times$ coverage) was carried out according to the Illumina standard sequencing method( $\mathrm{J}$ et al. 2014). The clean data for GWAS was obtained by removing the splices and inferior quality reads in the raw data. Clean reads were mapping to Rice Annotation version of 7.0 of accession Nipponbare from Michigan State University (http://rice.plantbiology.msu.edu/cgi-bin/gbrowse/rice/) with the BWA-MEM algorithm(Li 2013). The BAM files were then sorted using SAMtools software, and only reads with q value less than $10^{-30}$ were retained(Rajeshwari and Sarkar 2005). Next, SNP was called using GATK UnifiedGenotyper (v3.4-46) (Van der Auwera GA et al. 2013). The SNPs marked "PASS" by VCFtools software were kept. Only SNPs with missing rate lower than 0.2 and minor allele frequency (MAF) higher than 0.05 were used for genome-wide association study (GWAS).

\section{GWAS}


GWAS for mesocotyl length of rice seedlings was conducted by using EMMAX (mixed linear model). The population structure matrix was obtained by EIGENSOFT (version 6.0.1) for Principal component analysis (PCA). In order to minimize the impact of strongly linked genomic regions on PCA analysis, SNPs for PCA analysis were filtered using PLINK parameter -indep-pairwise $505 \mathrm{r}$. Kinship matrix was obtained by emmax-kin-intel tool in EMMAX software. Bonferroni correction method was used to correct the $p$ values of each SNP loci obtained from the association analysis, and a threshold of 0.05 was used to screen the significantly correlated SNP loci.

\section{Abbreviations}

QTL: Quantitative trait loci; CV: Coefficient of variation; GWAS: Genome-wide association study; SNP: Single nucleotide polymorphism; ANOVA: The analysis of variance; ML: Mesocotyl length.

\section{Declarations}

Ethical Approval and Consent to participate

Not applicable

\section{Consent for publication}

Not applicable

\section{Availability of supporting data}

The datasets supporting the conclusions of this article are included within the article and its additional files.

\section{Competing interests}

The authors declare that they have no competing interests.

\section{Funding}

This work was supported by funds from the Rice Molecular Design Breeding(2016YFD0101801).

\section{Authors' contributions}

$\mathrm{CHY}, \mathrm{SGL}$ and YPW conceived and designed the experiments. XJT, QG, YHL, YL, and HW performed the experiments. CHY, HW, PQ, BT, WLC, HY, BTM, XXL, CZL, SGL and YPW analyzed the data. HW wrote the manuscript. All authors have read and approved the final manuscript.

\section{Acknowledgements}

Not applicable 


\section{References}

1. Allan RE, Vogel OA, Burleigh JR, Peterson CJ (1961) Inheritance of Coleoptile Length and Its Association with Culm Length in Four Winter Wheat Crosses1 Crop Sci 1:328-332

2. Chen X et al. (2018) Research Advances of Rice Mechanical Direct-seeding Technology in China China Rice

3. Chen Y, Hao X, Cao J (2014) Small auxin upregulated RNA (SAUR) gene family in maize: Identification, evolution, and its phylogenetic comparison with Arabidopsis, rice, and sorghum $\mathrm{J}$ Integr Plant Biol 56:133-150

4. Dilday RH, Mgonja MA, Amonsilpa SA, Collins FC, Wells BR (1990) Plant height vs. mesocotyl and coleoptile elongation in rice: linkage or pleiotropism? Crop Sci 30:815-818

5. Farooq M, Siddique KHM, Rehman H, Aziz T, Lee D, Wahid A (2011) Rice direct seeding: Experiences, challenges and opportunities Soil and Tillage Research 111:87-98 doi:10.1016/j.still.2010.10.008

6. Feng F et al. (2017) Dynamic transcriptome and phytohormone profiling along the time of light exposure in the mesocotyl of rice seedling Sci Rep-Uk 7:11961

7. Gao S, Xie X, Chen Z, Wang X (2011) CHANGES OF ABA LEVEL AND EXPRESSION OF RELATED GENES DURING BLUE-LIGHT-REPRESSED MESOCOTYL ELONGATION IN har1 Journal of South China Normal University

8. Gao S, Xie X, Yang S, Chen Z, Wang X (2012) The changes of GA level and signaling are involved in the regulation of mesocotyl elongation during blue light mediated de-etiolation in Sorghum bicolor Mol Biol Rep 39:4091-4100

9. GER1, a GDSL Motif-Encoding Gene from Rice is a Novel Early Light- and Jasmonate-Induced Gene GER1, a GDSL Motif-Encoding Gene from Rice is a Novel Early Light- and Jasmonate-Induced Gene Plant Biology 9:32-40

10. Hande AS et al. (2017) Transcript profiling of genes expressed during fibre development in diploid cotton (Gossypium arboreum L.) Bmc Genomics 18:675

11. Hu Z et al. (2010) Strigolactones Negatively Regulate Mesocotyl Elongation in Rice during Germination and Growth in Darkness Plant \& Cell Physiology 51:1136-1142

12. Huizhen $\mathrm{H}$ et al. (2018) AtCSLD3 and GhCSLD3 mediate root growth and cell elongation downstream of the ethylene response pathway in Arabidopsis J Exp Bot:1065-1080

13. Hur YS, Shin KH, Kim S, Nam KH, Lee MS, Chun JY, Cheon Cl (2009) Overexpression of GmAKR1, a stress-induced aldo/keto reductase from soybean, retards nodule development Molecules \& Cells 27:217-223

14. J Z, K K, T F, A S (2014) PEAR: a fast and accurate Illumina Paired-End reAd mergeR. Bioinformatics (Oxford, England) 30:614-620 doi:10.1093/bioinformatics/btt593

15. Jie L, Zhou JJ, Zhang JZ (2018) Aux/IAA Gene Family in Plants: Molecular Structure, Regulation, and Function 
16. Kant S, Rothstein S (2009) Auxin-responsive SAUR39 gene modulates auxin level in rice Plant Signaling \& Behavior 4:1174-1175

17. Kondo N, Fujii T, Yamaki T (2010) Effect of light on auxin transport and elongation of Avena mesocotyl. Dev Growth Differ 11:46-61

18. Kumar V, Ladha JK (2011) Chapter Six - Direct Seeding of Rice : Recent Developments and Future Research Needs Adv Agron 111:297-413

19. Kutschera U, Wang ZY (2016) Growth-limiting proteins in maize coleoptiles and the auxinbrassinosteroid hypothesis of mesocotyl elongation Protoplasma 253:3-14

20. Lee H, Sasaki K, Higashitani A, Ahn S, Sato T (2012) Mapping and characterization of quantitative trait loci for mesocotyl elongation in rice (Oryza sativa L.) Rice 5:1-10 doi:10.1186/1939-8433-5-13

21. Lee H, Sasaki K, Kang J, Sato T, Song W, Ahn S (2017) Mesocotyl Elongation is Essential for Seedling Emergence Under Deep-Seeding Condition in Rice Rice 10 doi:10.1186/s12284-017-0173-2

22. Li H (2013) Aligning sequence reads, clone sequences and assembly contigs with BWA-MEM Genomics

23. Li H, Zhou S, Zhao W, Peng SS (2009) A novel wall-associated receptor-like protein kinase gene, OsWAK1 , plays important roles in rice blast disease resistance Plant Mol Biol

24. Li Y. (2016). Study on Influence of Light and Temperature and Bulked Segregation Analysis for Mesocotyl Elongation in Rice. master, Shanghai ocean university

25. Nakamura A, Umemura I, Gomi K, Hasegawa Y, Kitano H, Sazuka T, Matsuoka M (2010) Production and characterization of auxin-insensitive rice by overexpression of a mutagenized rice IAA protein Plant J 46:297-306

26. Niek, Stortenbeker, Marian, Bemer (2018) The SAUR gene family: the plant's toolbox for adaptation of growth and development. J Exp Bot

27. Ohno $\mathrm{H}$ et al. (2018) Longer mesocotyl contributes to quick seedling establishment, improved root anchorage, and early vigor of deep-sown rice Field Crop Res 228:84-92 doi:10.1016/j.fcr.2018.08.015

28. Purchase JL, Roux JL, Tonder HAV (1992) The effects of various seed treatments on the germination, coleoptile length and emergence of South African winter wheats (Triticum aestivum L.) South African Journal of Plant \& Soil 9:5

29. Qi G et al. (2013) Two poplar cellulose synthase-like D genes, PdCSLD5 and PdCSLD6, are functionally conserved with Arabidopsis CSLD3 J Plant Physiol 170:1267-1276

30. Rajeshwari G, Sarkar S. (SAM: A tool for software architecture modeling \& performance analysisQuantitative Evaluation of Systems, 2005. Second International Conference on the, 2005

31. Redoña ED, Mackill DJ (1996) Genetic Variation for Seedling Vigor Traits in Rice Crop Sci 36:285-290

32. S Marasini TNJL (2016) DIRECT SEEDED RICE CULTIVATION METHOD: A NEW TECHNOLOGY FOR CLIMATE CHANGE AND FOOD SECURITY The Journal of Agriculture and Environment

33. Sansen K, Wongboon W, Jairin J, Kato Y (2019) Farmer-participatory evaluation of mechanized dry direct-seeding technology for rice in northeastern Thailand Plant Prod Sci 22:46-53 
doi:10.1080/1343943X.2018.1557530

34. Shahi KMSA, RAU PB (2015) Popularising Direct Seeded Rice: Issues and Extension Strategies Online at https://mpra.ub.uni-muenchen.de/66486/

35. Shibo Zhang CCLL (2005) Evolutionary expansion, gene structure, and expression of the rice wallassociated kinase gene family. Plant Physiol 139:1107-1124

36. Slabaugh E, Held M, Brandizzi F (2011) Control of root hair development in Arabidopsis thaliana by an endoplasmic reticulum anchored member of the R2R3-MYB transcription factor family The Plant Journal

37. Soriano JB, Wani SP, Rao AN, Sawargaonkar GL, Gowda JAC (2018) Comparative Evaluation of Direct Dry-Seeded and Transplanted Rice in the Dry Zone of Karnataka, India Philippine Journal of Science 147

38. Spartz AK et al. (2012) The SAUR19 subfamily of SMALL AUXIN UP RNA genes promote cell expansion Plant $\mathrm{J} 70$

39. Turner FT, Chen CC, Bollich CN (1982) Coleoptile and Mesocotyl Lengths in Semidwarf Rice Seedlings1 Crop Sci 22:43-46

40. Van der Auwera GA et al. (2013) From FastQ data to high confidence variant calls: the Genome Analysis Toolkit best practices pipeline. Current protocols in bioinformatics 43:10-11 doi:10.1002/0471250953.bi1110s43

41. Wang D et al. (2007) Genome-wide analysis of the auxin response factors (ARF) gene family in rice (Oryza sativa). Gene 394:13-24

42. Watanabe H, Takahashi K, Saigusa M (2001) Morphological and anatomical effects of abscisic acid (ABA) and fluridone (FLU) on the growth of rice mesocotyls Plant Growth Regul 34:273-275

43. Weerakoon WMW, Mutunayake MMP, Bandara C, Rao AN, Bhandari DC, Ladha JK (2011) Directseeded rice culture in Sri Lanka: Lessons from farmers Field Crop Res 121:53-63

44. Wei et al. (2014) Cloning and characterization of the Oryza sativa wall-associated kinase gene OsWAK11 and its transcriptional response to abiotic stresses Plant \& Soil

45. Wu J et al. (2015) Genome-wide Association Study (GWAS) of mesocotyl elongation based on resequencing approach in rice Bmc Plant Biol 15 doi:10.1186/s12870-015-0608-0

46. Xihua L et al. (2017) A genome-wide analysis of the small auxin-up RNA (SAUR) gene family in cotton Bmc Genomics 18:815

47. Xiong Q et al. (2017) Ethylene-Inhibited Jasmonic Acid Biosynthesis Promotes Mesocotyl/Coleoptile Elongation of Etiolated Rice Seedlings Plant Cell:1053-1072

48. Yalan L. (2016). Study on Influence of Light and Temperature and Bulked Segregation Analysis for Mesocotyl Elongation in Rice. [M], Shanghai ocean university

49. Zhao Y et al. (2018) Genetic Architecture and Candidate Genes for Deep-Sowing Tolerance in Rice Revealed by Non-syn GWAS Front Plant Sci 9 doi:10.3389/fpls.2018.00332 


\section{Tables}

Due to technical limitations, table xlsx is only available as a download in the Supplemental Files section.

\section{Figures}

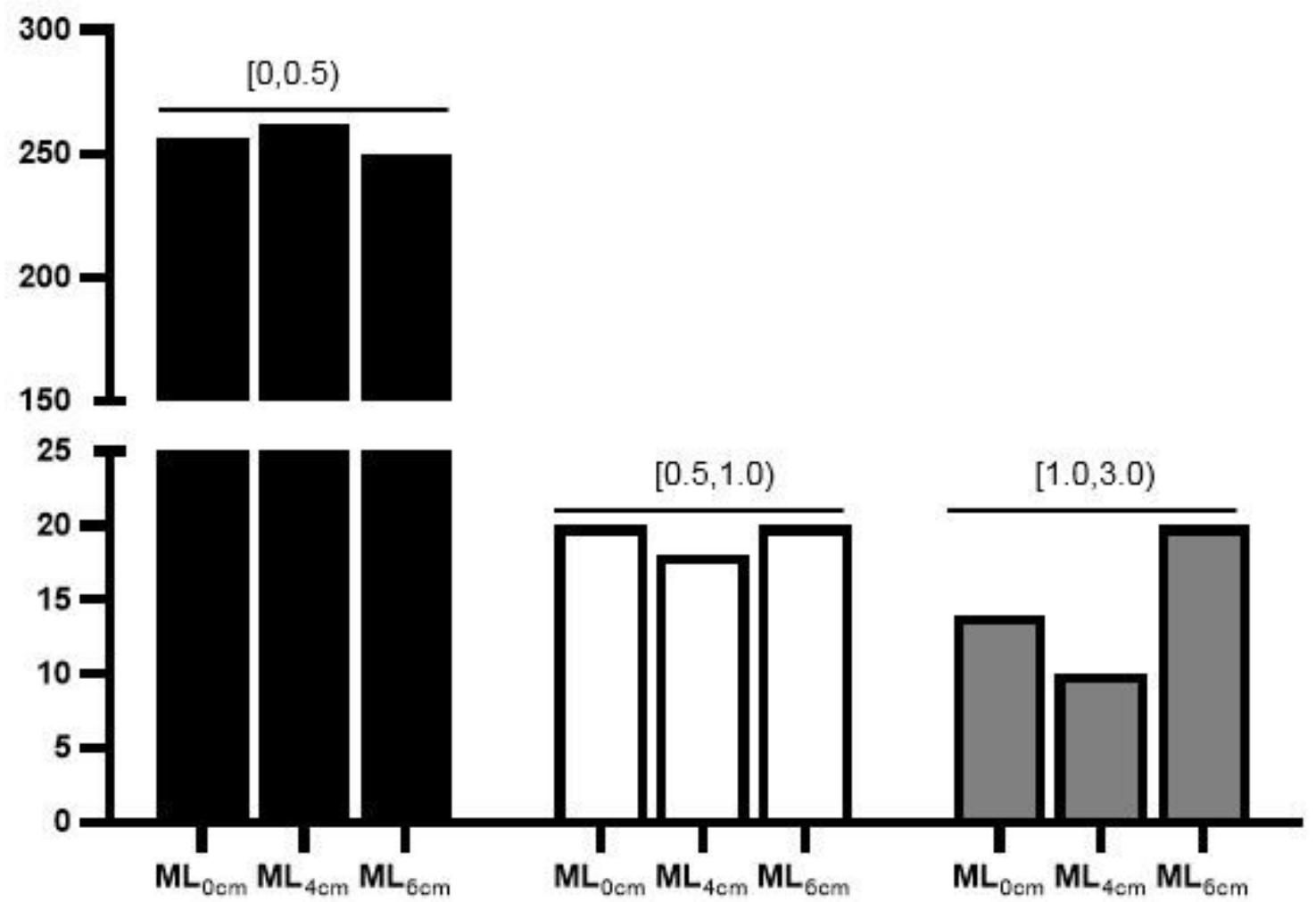

Figure 1

The mesocotyl length of 290 rice accesssions were classified under three different sowing depth. The group in black border and black fill is the number of rice variety whose mesocotyl length between $0-0.5 \mathrm{~cm}$ in three different sowing conditions; the group in black border and white fill is the number of rice variety whose mesocotyl length between $0.5-1.0 \mathrm{~cm}$ in three different sowing conditions; and the group in black border and gray fill is the number of rice variety whose mesocotyl length between $1.0-3.0 \mathrm{~cm}$ in three different sowing conditions. 
a

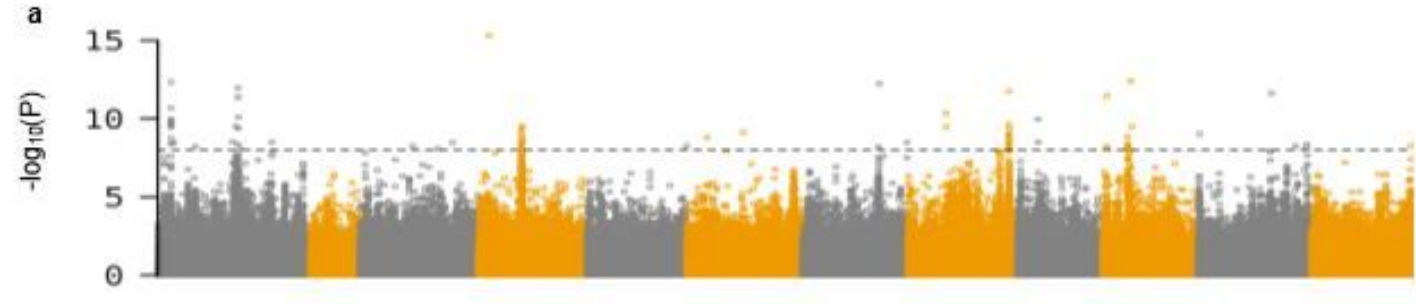

b

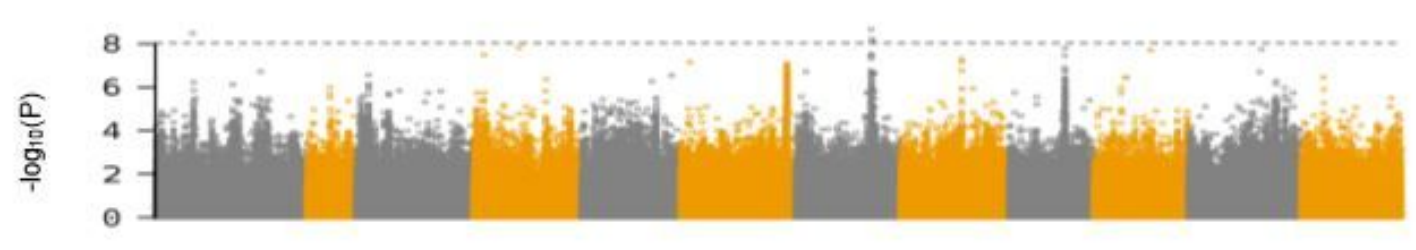

C

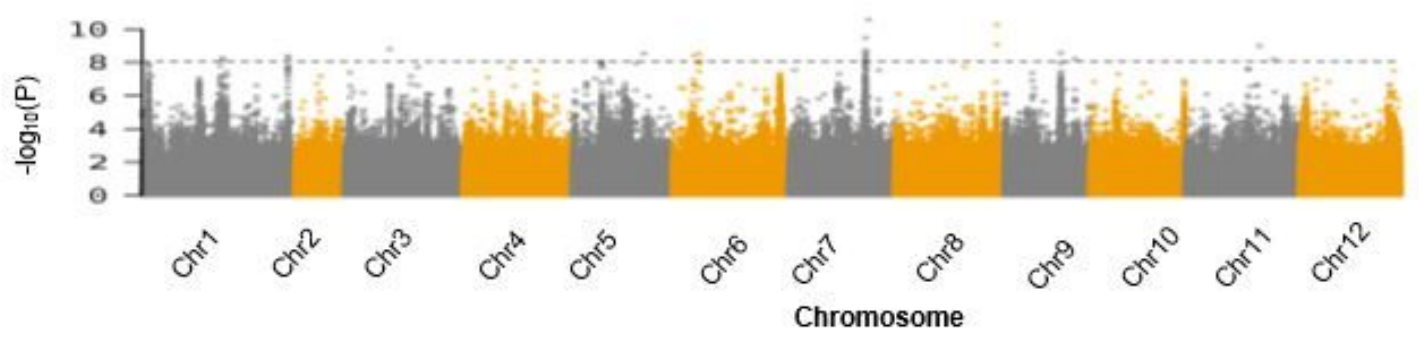

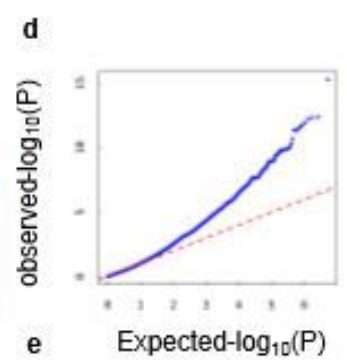

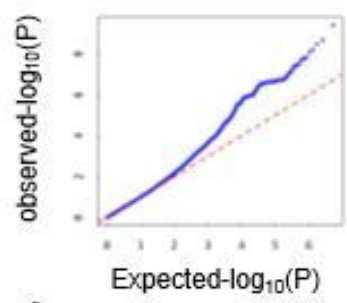

f

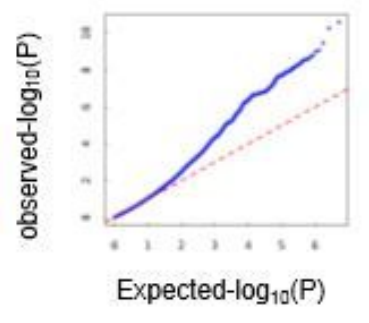

Figure 2

Manhattan plots of GWAS mapping for mesocotyl lengths. Manhattan plots of GWAS mapping for mesocotyl lengths measured in $0 \mathrm{~cm}$ sand culture $(\mathrm{MLO} \mathrm{cm}, \mathrm{a})$ in dark germination and in $4 \mathrm{~cm}, 6 \mathrm{~cm}$ sand culture (ML4cm,b, ML6cm,c) and Quantile-Quantile plots for (MLOcm ,d) , (ML4cm,e ) and (ML6cm,f). 


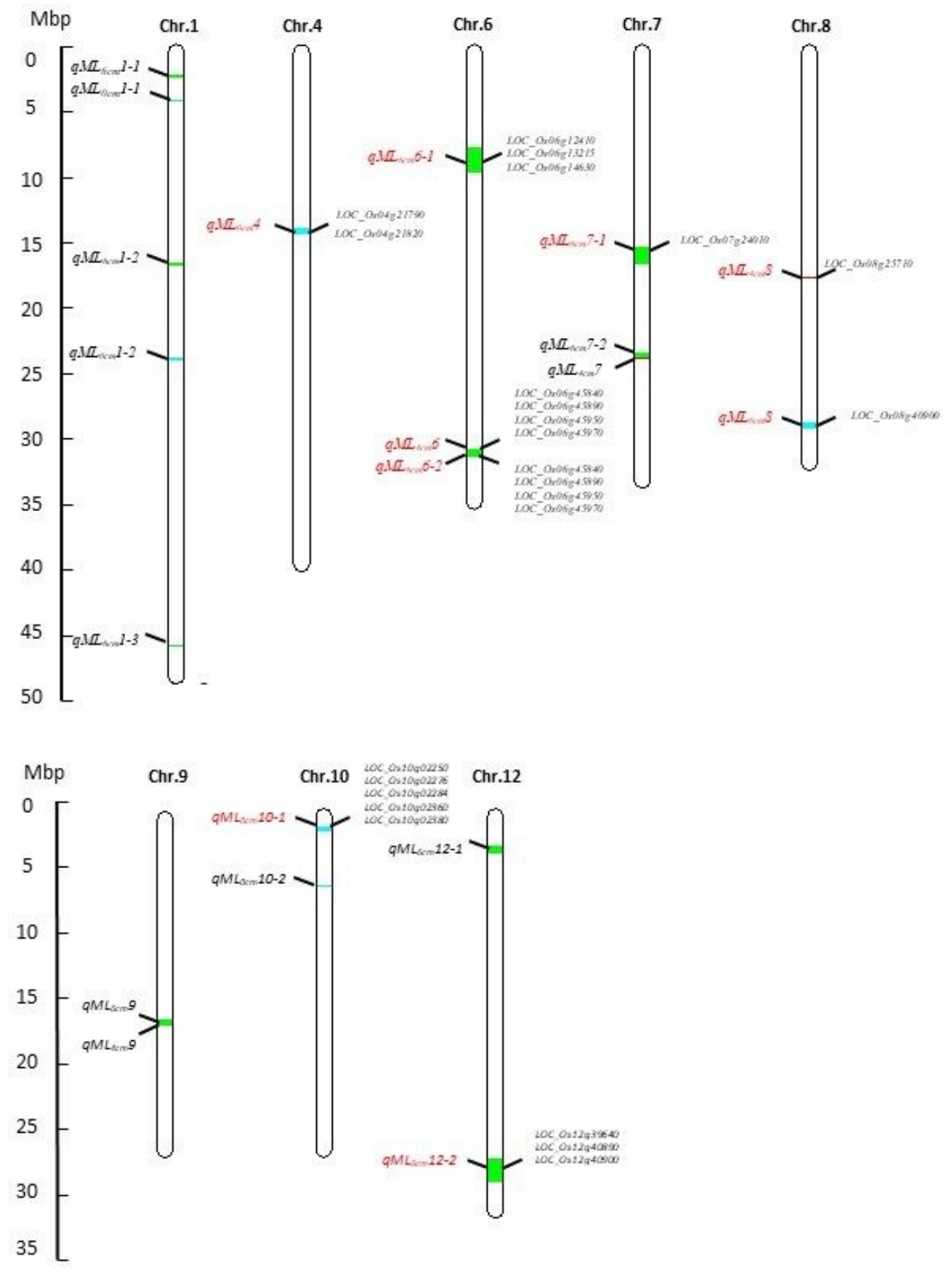

\section{Figure 3}

QTLs chromosome distribution of mesocotyl length in rice seedlings.

\section{Supplementary Files}

This is a list of supplementary files associated with this preprint. Click to download. 
- Tablesofmanuscript.xlsx

- Additionalfile1.xIsx 